\title{
Alendronate versus Raloxifene for Postmenopausal Women: A Meta-Analysis of Seven Head-to-Head Randomized Controlled Trials
}

\author{
Tiao Lin, ${ }^{1,2}$ Shi-Gui Yan, ${ }^{1}$ Xun-Zi Cai, ${ }^{1}$ Zhi-Min Ying, ${ }^{1}$ Fu-Zhen Yuan, ${ }^{1}$ and Xi Zuo ${ }^{3}$ \\ ${ }^{1}$ Department of Orthopaedic Surgery, Second Affiliated Hospital, School of Medicine, Zhejiang University, \\ Hangzhou, Zhejiang 310009, China \\ ${ }^{2}$ Department of Orthopaedic Surgery, Perelman School of Medicine, University of Pennsylvania, Philadelphia, PA 19014, USA \\ ${ }^{3}$ Department of Epidemiology and Biostatistics, School of Public Health, Drexel University, Philadelphia, PA 19102, USA
}

Correspondence should be addressed to Xun-Zi Cai; emilcai@hotmail.com

Received 14 May 2013; Accepted 31 October 2013; Published 5 May 2014

Academic Editor: Faustino R. Pérez-López

Copyright (C) 2014 Tiao Lin et al. This is an open access article distributed under the Creative Commons Attribution License, which permits unrestricted use, distribution, and reproduction in any medium, provided the original work is properly cited.

Purpose. The aim of this study was to directly compare the efficacy and the safety of the two agents for postmenopausal women. Methods/Principal Findings. Electronic databases were searched for relevant articles that met our predefined inclusion criteria. Seven randomized controlled trials (RCTs) involving 4054 women were identified and included. Although Aln was more effective than $\mathrm{Rlx}$ in increasing bone mineral density (BMD), no statistical differences were observed in reducing the risk of neither vertebral fractures $(P=0.45)$ nor nonvertebral fractures $(P=0.87)$ up to two-year followup. Aln reduced the risk of vasomotor $(P=0.006)$ but increased the risk of diarrhea compared to $\mathrm{Rlx}(P=0.01)$. Our subgroup analysis further indicated the difference between Aln and Rlx in fracture risk and was not materially altered by the administration pattern, the age. The weekly strategy of Aln would further reduce the upper gastrointestinal (GI) disorders and might gain more bone mass increment at lumbar spine compared to its daily treatment. Conclusion. There was no evidence of difference of fracture risk reduction between Aln and Rlx. In addition, age did not obviously influence their relative antifracture efficacy. For Aln the weekly strategy would further reduce the upper GI disorders and gain more bone mass increment compared to the daily treatment. During clinical decision making, the patients' adherence and the related side-effects associated with both drugs should also be taken into account.

\section{Introduction}

Osteoporotic fracture is a world-wide concern in the current aged society. It is estimated that annually there are 180,000 people encountering osteoporosis-related fractures in England and Wales. Postmenopausal women with bone loss were considered at high risk of bone fractures, which greatly impaired their life quality and led to mortality [1]. An appropriate and timely management for preventing osteoporotic fracture is extremely important. At present, antiresorptive agents are still the major treatments. Besides the novel Denosumab, which is a human monoclonal antibody of receptor activator of NF- $\kappa$ B ligand (RANKL) and potently suppresses osteoclastic bone resorption, alendronate (Aln), the most widely prescribed bisphosphonates, and raloxifene
(Rlx), the only Food and Drug Administration approved selective estrogen receptor modulators (SERMs), are the most evident antiresorptive agents for prevention and treatment of postmenopausal osteoporosis $[2,3]$.

For deciding the therapeutic strategy, it is highly imperative to know an estimate of the difference in fracture risk reduction between Aln and Rlx $[4,5]$. Although both therapies have established efficacy from randomized controlled trials (RCTs), Rlx was suggested to be less effective compared to Aln, mainly in preventing nonvertebral fractures $[3,6-8]$ and was therefore not recommended as a first-line treatment option for this population or considered as an alternative for young women with lower nonvertebral risk $[3,9]$.

However, so far the efficacy inferiority of Rlx under Aln for postmenopausal women, especially the most relevant 
outcome fractures prevention, was still inconclusive. (a) The evidence was mainly derived from the indirect comparison with placebo, of which the inherent defects should be respected $[10,11]$. With the statistical methods of indirect comparison such as the Bayesian method and the network meta-analysis $[6,12,13]$, there were significant inconsistencies in patient characteristics across the included studies. In particular, the population of the previous systematic reviews and meta-analyses were consisted of elderly osteoporotic men and patients with glucocorticosteroid-induced osteoporosis apart from postmenopausal women $[6,8,12]$. In addition, the adverse effects (AEs) of two agents, which would highly provide reference during clinical decision making, were not thoroughly compared in previous meta-analyses. (b) Recently, a large-scaled retrospective study conducted by Foster et al. [14] emphasized that after adherent treatment there was a similar risk reduction for the both drugs in both vertebral and nonvertebral fractures of women up to 8 years, which was inconsistent with the previous prospective evidence $[6,12,13]$. (c) There were emerging head-to-head RCTs to evaluate the comparative effectiveness of the two agents, the results of which were mainly limited by the small sample size but those available comparative data should be well summarized and taken into consideration [15-21].

Taken together, this meta-analysis with all the available 7 head-to-head RCTs involving 4054 participants was conducted to summarize the comparative efficacy of bone mass increment and fracture prevention between Aln and Rlx for postmenopausal women [15-21]. Their safety profiles were also reviewed in a head-to-head comparative manner. Besides, we aimed to evaluate clinically-related and designrelated factors which might contribute to the difference in efficacy and AEs.

\section{Methods}

2.1. Literature Search. Electronic databases (PubMed, Medline, EMBASE, Clinical Trial Registry and the Cochrane Data Base of Systematic Reviews, and the Cochrane Central Register of Controlled Trials) were searched without limit by two independent investigators (Lin and Ying), which were last updated on October, 2013. The search used terms and Boolean operators as follows: "(alendronate OR bisphosphonate) AND (raloxifene OR selective estrogen receptor modulators) AND postmenopausal women AND (osteoporosis OR fracture)." Reference lists of all the selected articles were hand-searched for any additional trials.

2.2. Identification of Eligible Studies. The trials were reviewed in which (a) the target population were consisted of postmenopausal women with low bone mass, (b) the interventions at least included both Aln and Rlx therapies, (c) the outcomes at least comprised one of the following assessments: fracture incidence, $\mathrm{BMD}$, or safety profile, and (d) the trials were randomized controlled trials (RCTs). The trials were excluded if (a) patients had a prior history of metastatic bone disease, (b) phase-I or observational studies, case reports, and reviews, and (c) the same RCTs were reanalyzed. Disagreements were resolved through discussions.

2.3. Assessment of Study Quality. Two reviewers (Lin and Ying) independently assessed the study validity with Cochrane Collaboration's tool for assessing the risk of bias, which addresses six specific domains such as sequence generation, allocation concealment, blinding, incomplete outcome data, and selective outcome reporting. Whether the included trials were similar in baseline, adopting similar cointerventions, and applying intention-to-treat (ITT) analysis was also evaluated. Disagreement was evaluated by means of kappa $(\kappa)$ test and resolved by discussion [22].

2.4. Data Abstraction, Conversion, and Analysis. For each eligible trial, two of us (Lin and Ying) independently extracted the relevant data and checked the accuracy. In particular, we abstracted study design, sample size, demographic data (age, body mass index, and baseline BMD), intervention protocol, duration of the trial, loss to followup, trial outcomes (fracture incidence, BMD, and incidence of adverse events), and industrial funding. We contacted the first or the corresponding author of each eligible trial to verify the accuracy of the data abstraction as well as our methodological assessment.

The overall incidences of vertebral or nonvertebral fractures (hip, upper leg, lower leg, pelvis, hummers, wrist/forearm, clavicle/rib, and other) in the two groups were our primary outcome. We also evaluated the BMD percentage changes from the baseline at lumbar spine (LS), femoral neck (FN), and total hip (TH) in both groups. BMD was measured by dual-energy X-ray absorptiometry (DXA). The safety profile comprised the reported discontinuations due to AEs, AEs probably related to Aln (upper gastrointestinal disorders (GI) and diarrhea), and AEs probably related to Rlx (vasomotor events and venous thrombosis).

We took fracture risk reduction, LS BMD, and risk of upper gastrointestinal (GI) disorders at the end of follow-up as our main meta-analysis on basis of their sufficient trials for subgroup analysis.

We preferentially used the ITT data from the trials whenever possible. If the data were not reported in the original article, we extrapolated them from the accompanying graphs. To maximize data availability, we applied percentage change data for BMD and serum lipid outcome. If percentage change data were unavailable in BMD outcome, we imputed the percentage change data using (endpoint data, baseline data) divided by baseline data then multiplying 100 times. For the missing standard errors (SEs) of BMD data, the maximum SEs extracted from Muscoso et al. [18] were conservatively chosen for all BMD percentage change SEs. The sensitivity analysis was performed through omitting trials with imputed SEs to assess the variation in overall effect [22].

The fracture incidence and the safety profile outcomes were presented as risk ratio (RR) with $95 \%$ confidence intervals (CI) and combined using the Mantel-Haenszel method. BMD were pooled with the inverse variance method and presented as weighted mean differences (WMD) and 95\% CI. We calculated the statistical heterogeneity using a Chi-squared 


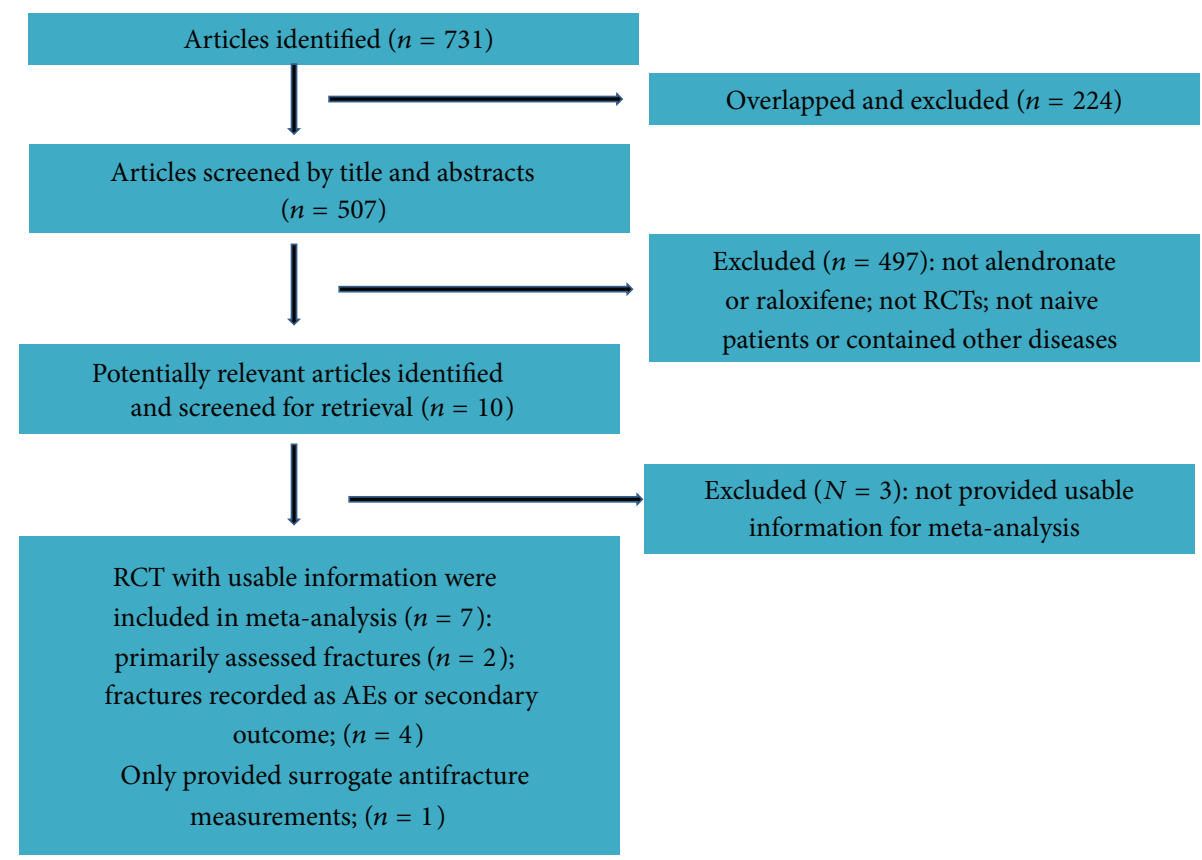

FIGURE 1: Flow chart for the meta-analysis.

$\left(\chi^{2}\right)$ test with the significance at 0.1 . We also assessed the inconsistency $I^{2}$ to describe the percentage of the variability in effect estimates due to the heterogeneity. We considered a value greater than $50 \%$ as the substantial heterogeneity. Fixed effects model would be applied if there were no statistical heterogeneity among the studies; otherwise, we used the random effects model. If substantial heterogeneities across studies $\left(I^{2}>50 \%\right)$ were detected in the index five main meta-analysis, we performed post hoc sensitivity analysis by omitting the outlier studies to determine the sources of Cochran's heterogeneity [22]. The outliers were detected as the studies with confidence interval of the estimated effect size were not well overlapping with the pooled overall effect size [23].

The subgroup analyses in the main meta-analysis were performed by baseline characteristics of the studies: patterns of treatments in Aln groups (daily or weekly), mean age of participants ( $>65$ or $\leqq 65)$, methodological quality, sample size $(\geqq 400$ or $<400)$, and industrial funding. BMI of participants and dose of agents could not be analyzed in subgroup analysis due to the difficulties in determining cutoff values. To determine the influence of outlier studies, after omitting the two detected outliers, the pooled-analysis and the subgroup analyses were repeated in the main analysis with statistical heterogeneities. Results of subgroup analysis were presented only if each subgroup comprised at least two trials.

To comprehensively identify the clinical-related modifiers, metaregression with covariates (age, BMI of participants, patterns of Aln administration) were carried out in the fracture (vertebral fracture analysis was not performed as only 3 trials included) and GI disorder analysis.

To evaluate the publication bias, we used Begg's test and Egger's test with trials from fracture outcomes analysis, including 6 trials in total fractures, 3 trials in vertebral fractures, and 4 trials in nonvertebral fractures [22].

Meta-analysis was conducted using Review Manager 5.1 software. Metaregression analysis, Begg's test, and Egger's test were performed through STATA 11.0 (Stata Corp, College Station, TX, USA). The criteria of the Grading of Recommendations Assessment, Development, and Evaluation (GRADE) were used to evaluate the quality of evidence by each outcome [24].

\section{Results}

3.1. Study Identification. Literature search initially yielded 731 relevant articles; of which 224 overlapped publications were excluded. From the remaining 507 articles, 497 were excluded since they did not fulfill the selection criteria based on their titles and abstracts. After full-text checking of the rest of 10 RCTs [15-21, 25-27], 3 RCTs were excluded as their outcomes did not meet the inclusion criteria [25-27]. Finally, 7 studies with usable information were included in our meta-analysis [15-21] (Figure 1). The weighted kappa for the agreement on eligibility between reviewers was 0.81 (95\% CI: $0.72-0.90$ ).

3.2. Study Characteristics. The characteristics of the included 7 trials were shown in Table 1 [15-21]. Four of the trials were double blinded and placebo-used, multicenter RCTs $[16,17,19,20]$. There were two studies designed with fractures as endpoint in a two-year followup [18, 19]. Other five studies $[15-17,20,21]$ with one-year followup used BMD as surrogates for antifracture assessment, and the fractures was reported as AEs $[16,17,20]$ or secondary outcomes $[15,18]$. Four studies only comprised Aln and Rlx treatments $[15,17,19,20]$. The other three studies contained combined 


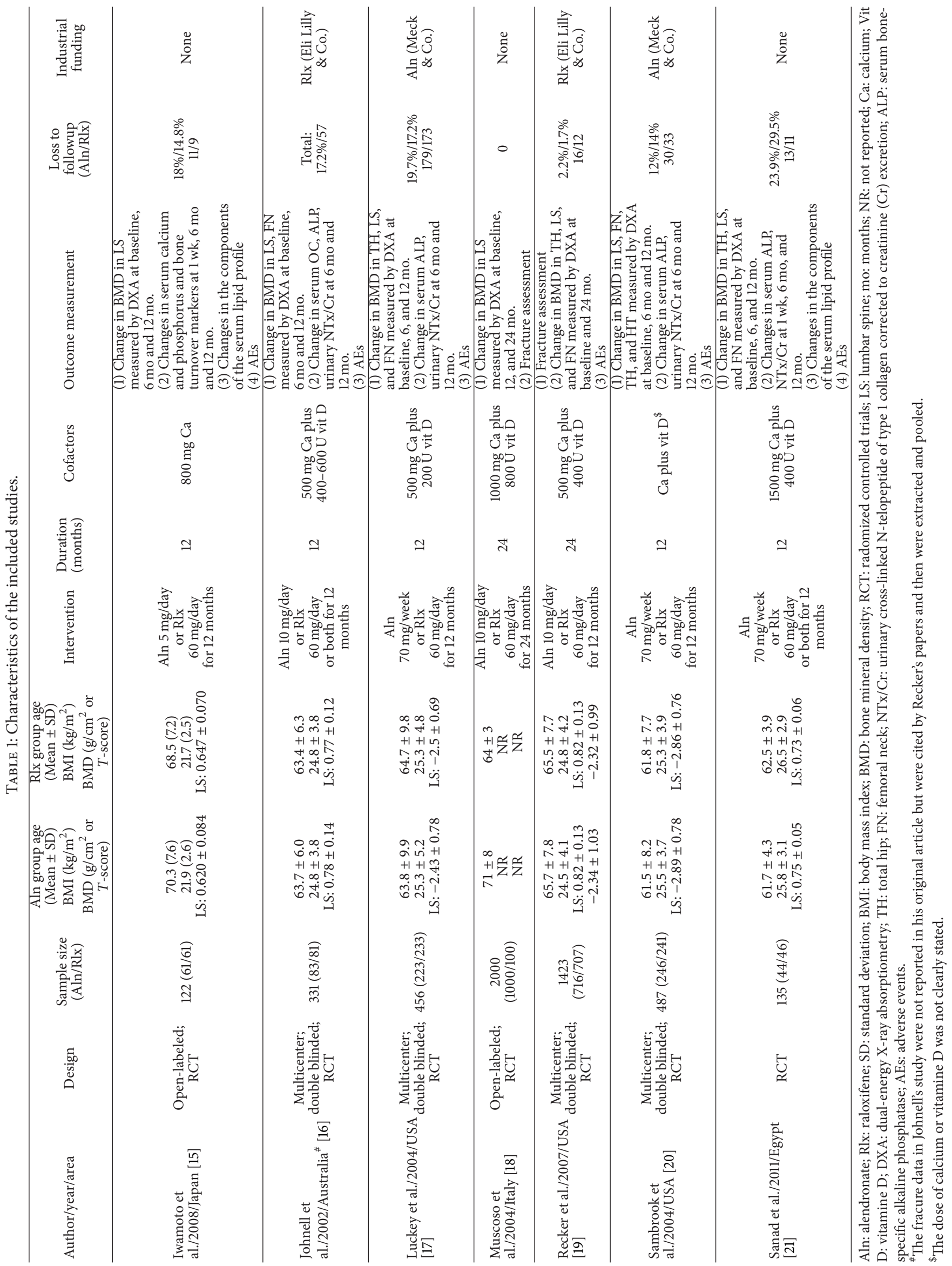




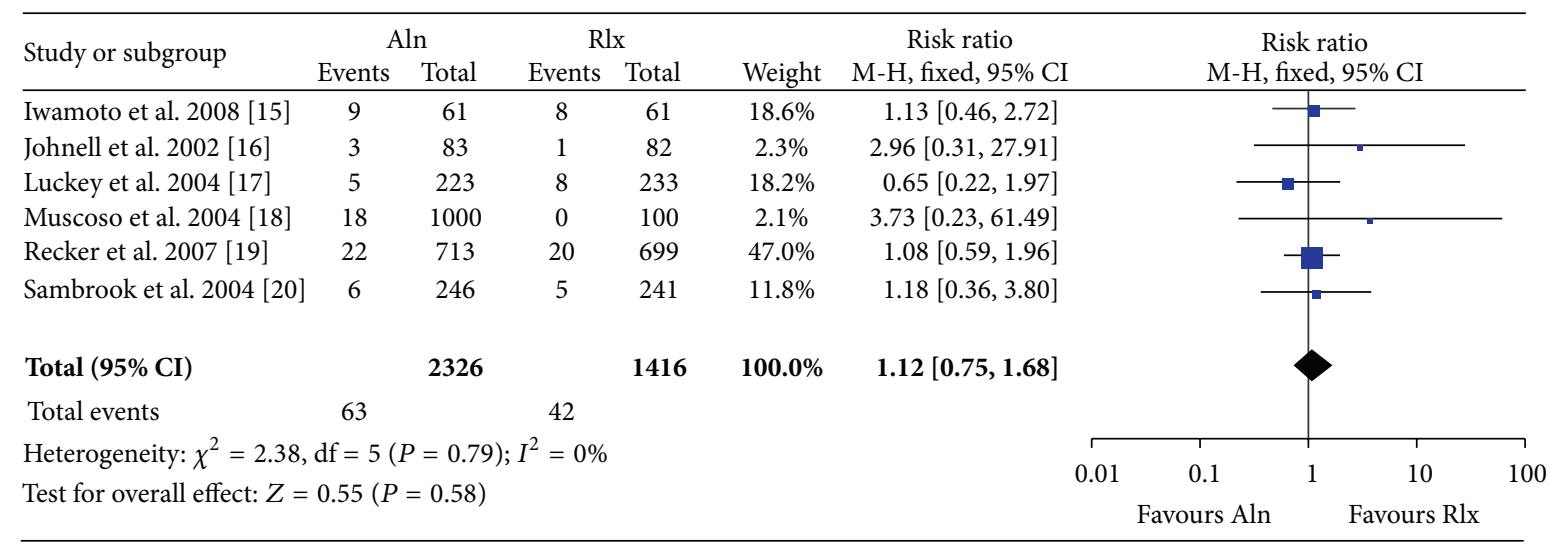

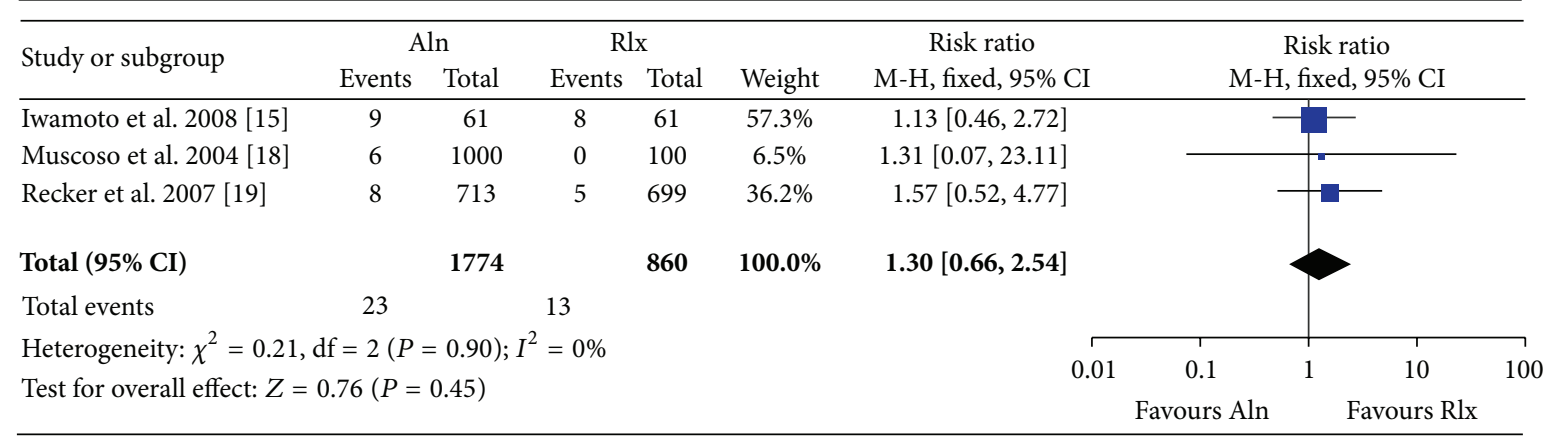

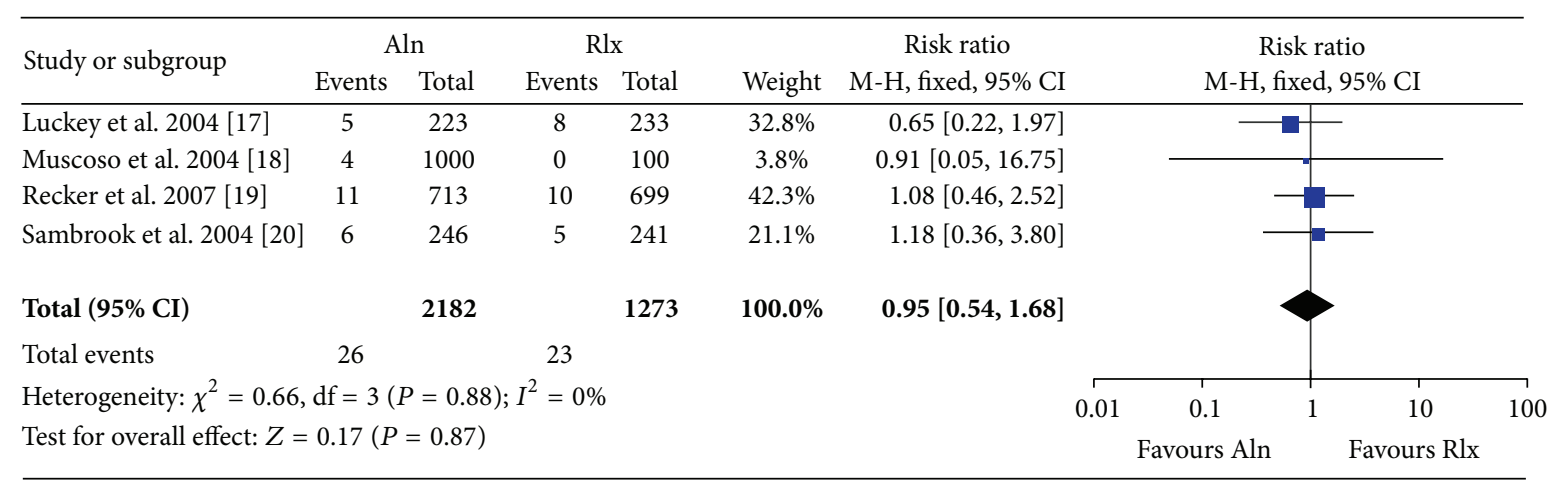

FIGURE 2: Relative risk of total fractures, vertebral fractures, and nonvertebral fractures for postmenopausal women between the alendronate group and the raloxifene group. NV: nonvertebral.

treatment $[16,21]$ or other therapies as well [18]. Only one trial was considered to have substantial loss to follow-up (more than 20\%) [21], while the rates were acceptable among the other studies (range, $1.7 \%$ to $19.7 \%$ ). Two studies were funded by Aln Company (Merck \& Co.) $[17,20]$ and two were funded by Rlx Company (Eli Lilly \& Co.) $[16,19]$, while the left three did not involve any industrial funding $[15,18,21]$. In terms of the patterns of administrations in Aln groups, four studies treated women once daily $[15,16,18,19]$, while the other three adopted once weekly strategy $[17,20,21]$. Patients in the both groups took calcium and vitamin $\mathrm{D}$ as supplementations equally in all eligible studies.

3.3. Study Quality. The methodological quality was evaluated independently by two reviewers (Lin and Ying) with Cochrane Collaboration's tool for assessing the risk of bias and showed in Table 2 [22]. Four trials $[6,7,9,10]$ described explicit adequately randomization, concealment of allocation assignment, proper blinding, and applying intention to treat analysis, which were low risk of bias $[16,17,19,20]$, while the other three trials with inexplicit randomization and inadequate blinding were considered moderate risk of bias $[15,18,21]$. The weighted kappa for the agreement on the trial quality between reviewers was $0.8495 \% \mathrm{CI}$ : $(0.75-$ $0.93)$.

3.4. Effect of Interventions. No differences in total, vertebral or nonvertebral fracture incidences were demonstrated between the Aln groups and Rlx groups. (Total: 6 studies, fixed-effects RR (95\% CI): $1.19(0.75,1.68), P=0.58, I^{2}=0 \%$; vertebral: 3 studies, fixed-effects RR (95\% CI): 1.30 (0.66 to 2.54), $P=0.45$, and $I^{2}=0 \%$; nonvertebral: 4 studies, fixedeffects RR (95\% CI): $0.95(0.54,1.68), P=0.87$, and $I^{2}=$ $0 \%$, Figure 2). Our meta-analysis indicated moderate quality 


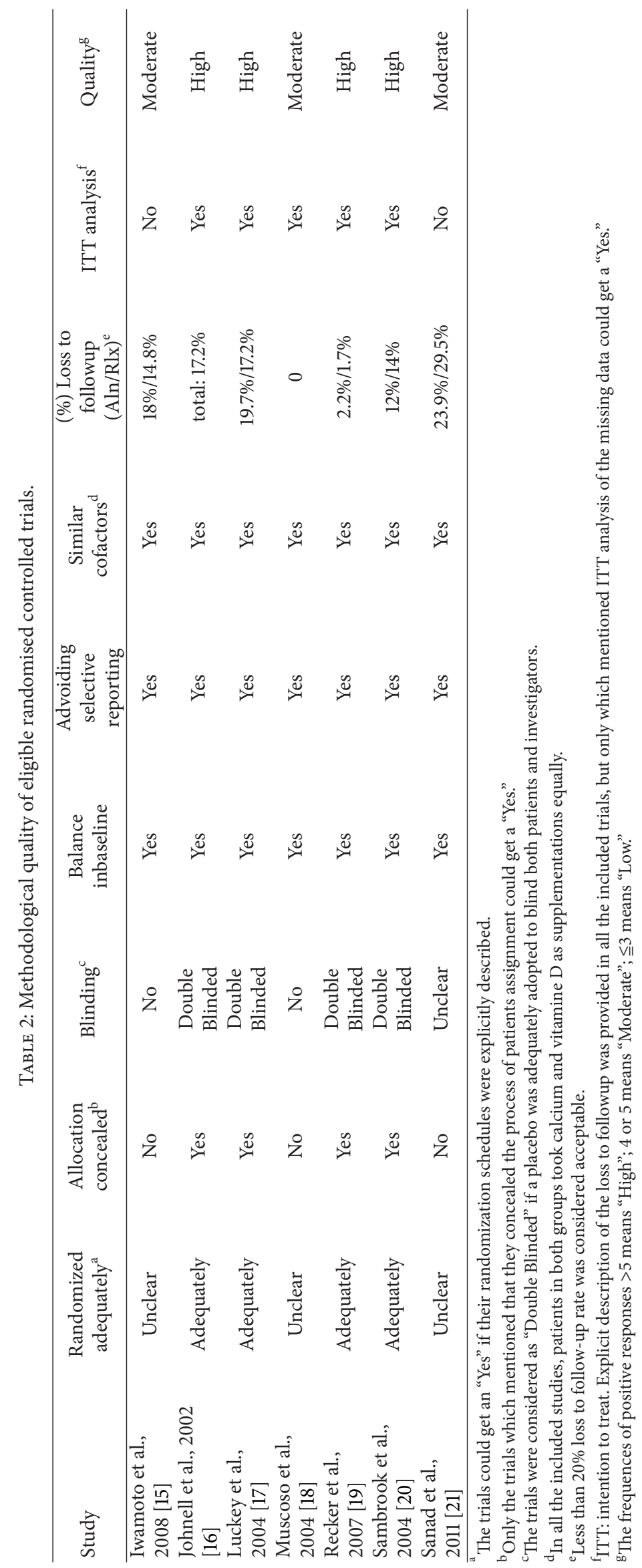


TABLE 3: ${ }^{\$}$ GRADE evidence profile: randomized controlled trials of comparison between Aln and Rlx for postmenopausal women.

\begin{tabular}{|c|c|c|c|c|c|c|c|}
\hline \multirow{2}{*}{$\begin{array}{l}\text { No. of trials } \\
\text { (No. of women) }\end{array}$} & Summary of & \multicolumn{6}{|c|}{ Quality of evidence } \\
\hline & $\begin{array}{l}\text { Magnitude of } \\
\text { effect ( } 95 \% \text { CI) }\end{array}$ & $\begin{array}{c}\text { Risk of } \\
\text { bias }\end{array}$ & Inconsistency & Indirectness & Imprecision & $\begin{array}{c}\text { Publication } \\
\text { bias }\end{array}$ & Quality \\
\hline
\end{tabular}

Antifracture evaluation

Total fractures risk

6 trials (3742) $\begin{gathered}\text { RR: } 1.12 \\ (0.75 \text { to } 168)\end{gathered}$ Low $\quad$ No $\quad$ Direct $\quad$ Yes ${ }^{c} \quad$ Unlikely Moderate

Vertebral fractures risk

3 trials (2634)

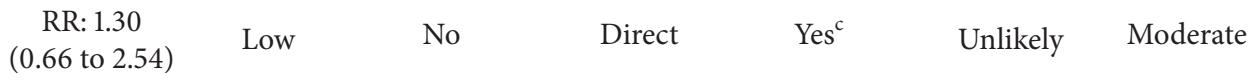

Nonvertebral fractures risk

4 trials (3455) $\begin{gathered}\text { RR: } 0.95 \\ (0.54 \text { to } 1.68)\end{gathered}$ Low $\quad$ No $\quad$ Direct $\quad$ Yes $^{\text {c }} \quad$ Unlikely Moderate

Surrogate anti-fracture evaluation

LS BMD at 12 months

\begin{tabular}{|c|c|c|c|c|c|c|c|}
\hline 6 trials $(2396)$ & $\begin{array}{c}\text { WMD: } 2.92 \\
\text { (2.23 to } 3.62)\end{array}$ & Low & Inconsistency ${ }^{\mathrm{b}}$ & Direct & No & Unlikely & Moderate \\
\hline 5 trials $(2274)^{*}$ & $\begin{array}{l}\text { WMD: } 2.37 \\
\text { (2.17 to } 2.58)\end{array}$ & Low & No & Direct & No & Unlikely & High \\
\hline
\end{tabular}

FN BMD at 12 months

4 trials (1174)

\begin{tabular}{|c|c|c|}
\hline $\begin{array}{l}\text { VMD: } 0.84 \\
0.32,1.36)\end{array}$ & Low & Inconsistency ${ }^{\mathrm{b}}$ \\
\hline
\end{tabular}

TH BMD at 12 months

$$
3 \text { trials (1009) }
$$

Direct

No

Unlikely

Moderate

$$
\text { Safety profile }
$$

Risk of upper GI events

$$
\begin{aligned}
& 6 \text { trials }(2708) \\
& 5 \text { trials }(2221)^{\#}
\end{aligned}
$$

Risk of discontinuations

5 trials (2642)

Risk of VT events

\begin{tabular}{|c|c|c|c|c|c|}
\hline $\begin{array}{c}\text { RR: } 1.10 \\
\text { (0.77 to } 1.58)\end{array}$ & Low & Inconsistency $^{\mathrm{b}}$ & No & $\mathrm{Yes}^{\mathrm{c}}$ & Unlikely \\
\hline $\begin{array}{c}\text { RR: } 1.30 \\
\text { (1.04 to } 1.63)\end{array}$ & Low & No & No & No & Unlikely \\
\hline
\end{tabular}

$$
3 \text { trials (1934) }
$$

Risk of diarrhea events

$$
3 \text { trials (1600) }
$$

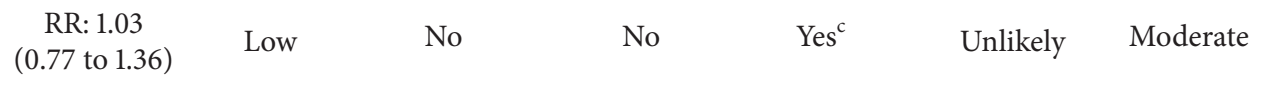

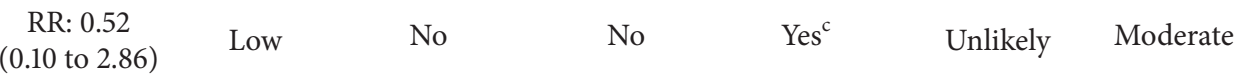

\begin{tabular}{ccccccc}
$\begin{array}{c}\text { RR: } 2.33 \\
(1.21 \text { to } 4.49)\end{array}$ & High $^{\text {a }}$ & No & No & No & Unlikely & Moderate \\
\hline
\end{tabular}


TABLE 3: Continued.

\begin{tabular}{|c|c|c|c|c|c|c|c|}
\hline \multirow{2}{*}{$\begin{array}{l}\text { No. of trials } \\
\text { (No. of women) }\end{array}$} & \multirow{2}{*}{$\begin{array}{l}\text { Summary of } \\
\text { finding } \\
\text { Magnitude of } \\
\text { effect ( } 95 \% \text { CI) }\end{array}$} & \multicolumn{6}{|c|}{ Quality of evidence } \\
\hline & & $\begin{array}{c}\text { Risk of } \\
\text { bias }\end{array}$ & Inconsistency & Indirectness & Imprecision & $\begin{array}{c}\text { Publication } \\
\text { bias }\end{array}$ & Quality \\
\hline \multicolumn{8}{|c|}{ Risk of vasomotor events } \\
\hline 2 trials $(943)$ & $\begin{array}{c}\text { RR: } 0.47 \\
(0.27 \text { to } 0.81)\end{array}$ & Low & No & No & No & Unlikely & High \\
\hline \multicolumn{8}{|c|}{ Risk of vasodilatation events } \\
\hline 3 trials $(1643)$ & $\begin{array}{c}\text { RR: } 0.74 \\
\text { (0.54 to } 1.01)\end{array}$ & Low & No & No & $\mathrm{Yes}^{\mathrm{c}}$ & Unlikely & Moderate \\
\hline \multicolumn{8}{|c|}{ 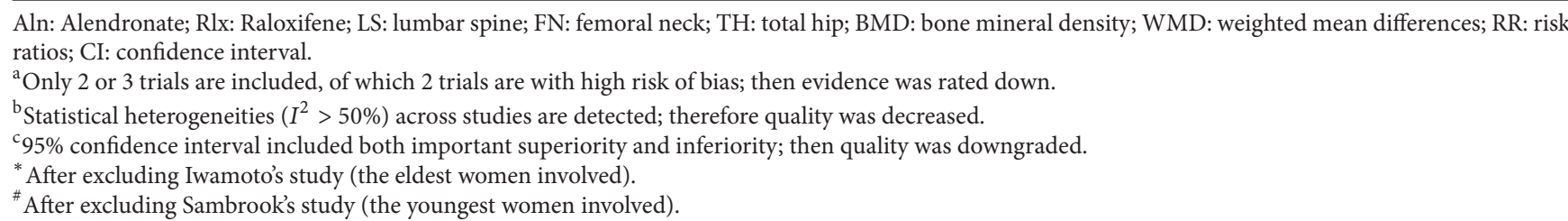 } \\
\hline
\end{tabular}

evidence of equivalent efficacies between the two medications in fractures prevention (Table 3).

All of the included studies reported BMD data measured by DXA at least at one skeleton site. Both Aln and Rlx increased BMD significantly at LS, FN, and TH after 6, 12, and 24 months related to the baseline. Aln obtained bone mass increment to a greater extent than Rlx (Table 4), and the differences were widening as the treatment continued. The evidence quality was moderate (Table 3 ).

Both Aln and Rlx were well tolerated, no fatal AEs related to treatment were reported. It was similar in drop out due to AEs, upper GI disorders, venous thrombosis, and vasodilatation in the both groups: (Aln versus Rlx: drop out due to AEs: 5 studies, RR: 1.03 (0.77 to 1.36$), P=0.85$, and $I^{2}=0 \%$; upper GI disorders: 6 studies, RR: 1.10 (0.77 to 1.58$)$, $P=0.60$, and $I^{2}=52 \%$; venous thrombosis: 3 studies, RR: 0.52 ( 0.10 to 2.86$), P=0.45$, and $I^{2}=0 \%$; vasodilatation: 3 studies, RR: 0.74 (0.54 to 1.01 ), $P=0.06$, and $I^{2}=0 \%$, Figure 3 ). And the evidence quality for the differences among those AEs risks was moderate to high with the exception for Aln increase greater risks of upper GI disorders than Rlx, which was supported by low quality evidence (the quality of evidence turned out to be high if excluding the outlier study [20]) (Table 3).

Moderate to high quality evidence showed that Aln would increase $133 \%$ risks of diarrhea while avoid 57\% risks of vasomotor events compared to Rlx (Aln versus Rlx: diarrhea: 3 studies, RR: 2.33 (1.21 to 4.49 ), $P=0.01$, and $I^{2}=0 \%$; vasomotor: 2 studies, RR: 0.47 (0.27 to 0.81$), P=0.006$, and $I^{2}=0 \%$, Figure 3 ).

3.5. Heterogeneity and Outlier. Of the main meta-analysis, substantial heterogeneities were detected in outcomes of LS BMD (at 12 months: $P<0.01, I^{2}=95 \%$ ) and upper GI disorders $\left(P=0.60, I^{2}=52 \%\right)$. In LS BMD comparison (at 12 months), Iwamoto' study was found as an outlier [15]. After omitting this study, the results showed insignificant heterogeneities across studies $\left(P=0.19, I^{2}=35 \%\right)$ and the estimate effect size (WMD) in LS BMD was only reduced from 2.92 (95\% CI: 2.23 to 3.62 ) to 2.37 (2.17 to 2.58). Sambrook's research turned out to be an outlier in the GI disorders [20]. The heterogeneities in GI disorders were simultaneously reduced to be minimal $\left(P=0.83, I^{2}=0 \%\right)$ after excluding this study while the differences between Aln and Rlx in risks of GI disorders turned out to be statistical (Aln versus Rlx: RR: $1.30(1.04,1.63), P=0.02)$. Since the two outlier studies were identified, the subgroup analysis were repeated after excludion of them in LS BMD at 12 months and GI disorders respectively.

3.6. Sensitivity Analysis and Subgroup Analysis. The overall results of main meta-analysis were not significantly altered by omitting trials with imputed SEs. Our subgroup analysis suggested that patterns of administrations in Aln groups, participants' age, methodological quality, sample size, or industrial funding of included studies were not associated with the overall effect size of the differences in fracture reduction. The outlier studies did not alter the results of the subgroup analysis in incidences of GI disorders $[15,20]$.

The higher risk of Aln in upper GI disorders compared to Rlx was detected in the subgroups containing studies with daily administrated Aln (Aln versus Rlx: RR: 1.34 (1.04, 1.72), $P=0.02$ ) and with participants over 65 years old (Aln versus Rlx: RR: $1.32(1.01,1.73), P=0.04)$, which remained unchanged either including or excluding Sambrook et al. [20]. Notwithstanding, after excluding Iwamoto's study [15], the studies involving weekly treated Aln groups contributed to a greater difference in LS bone gain between Aln and Rlx groups compared to those which adopted daily strategies in Aln groups (weekly versus daily: WMD difference: 0.36, 


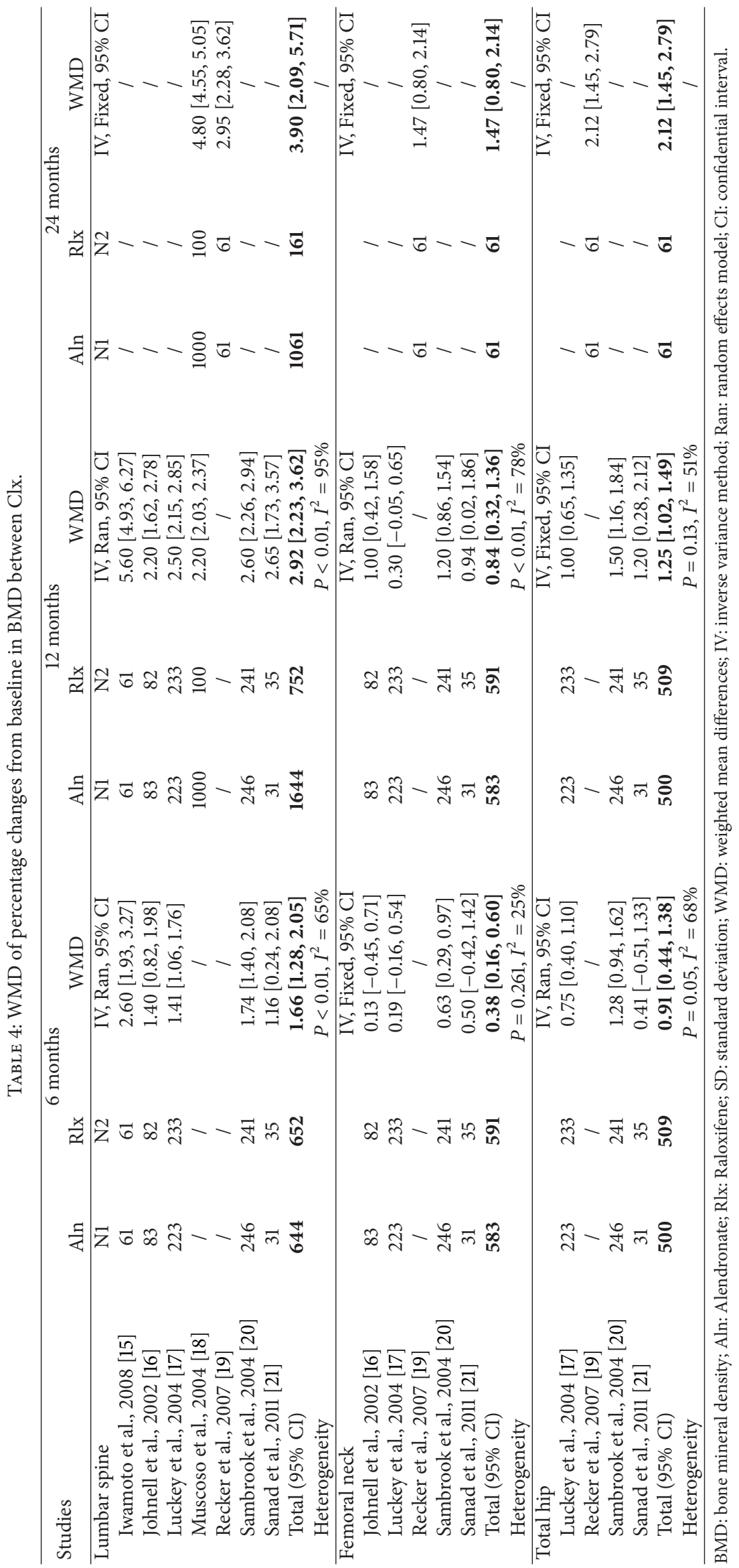




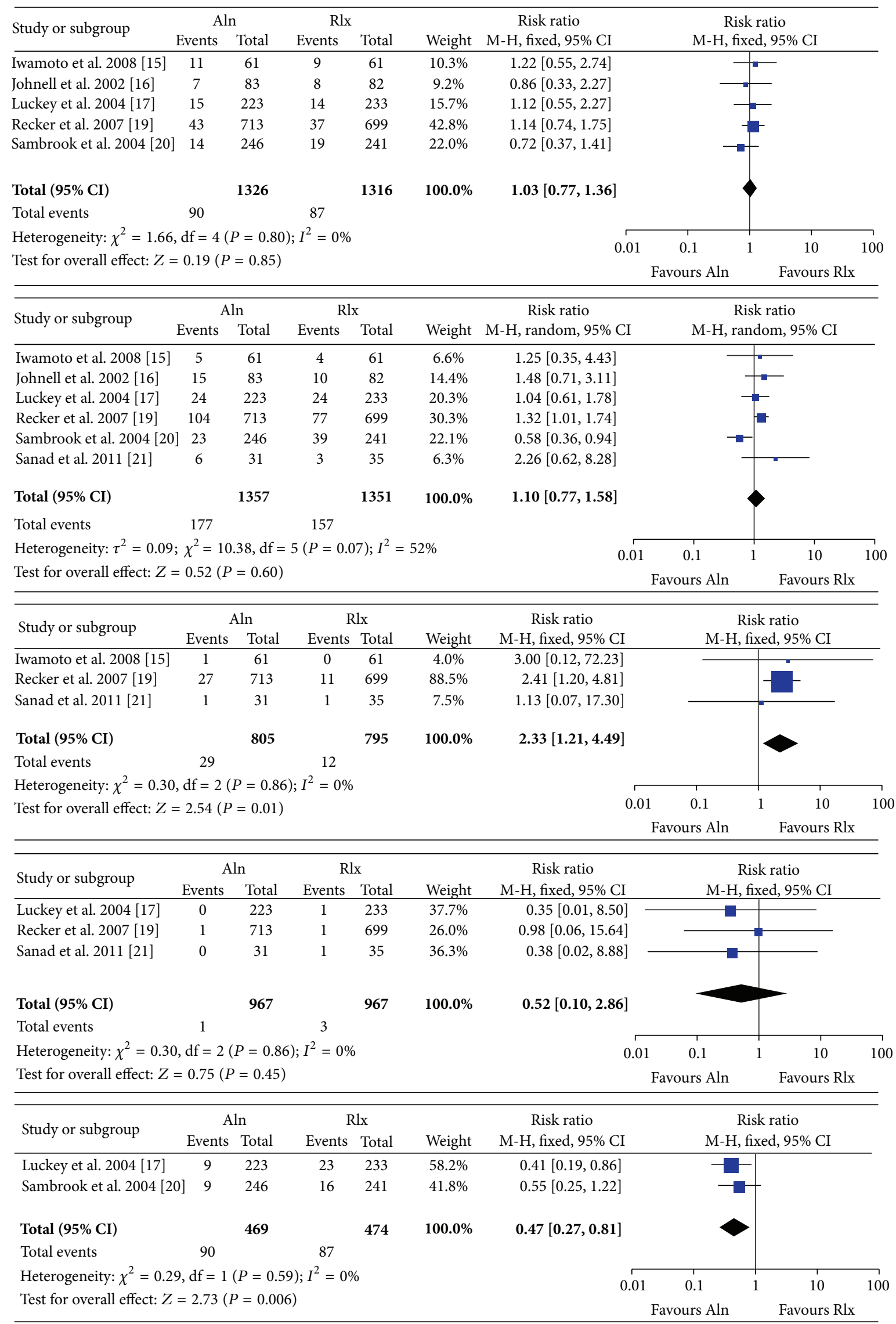

(a)

FIGURE 3: Continued. 


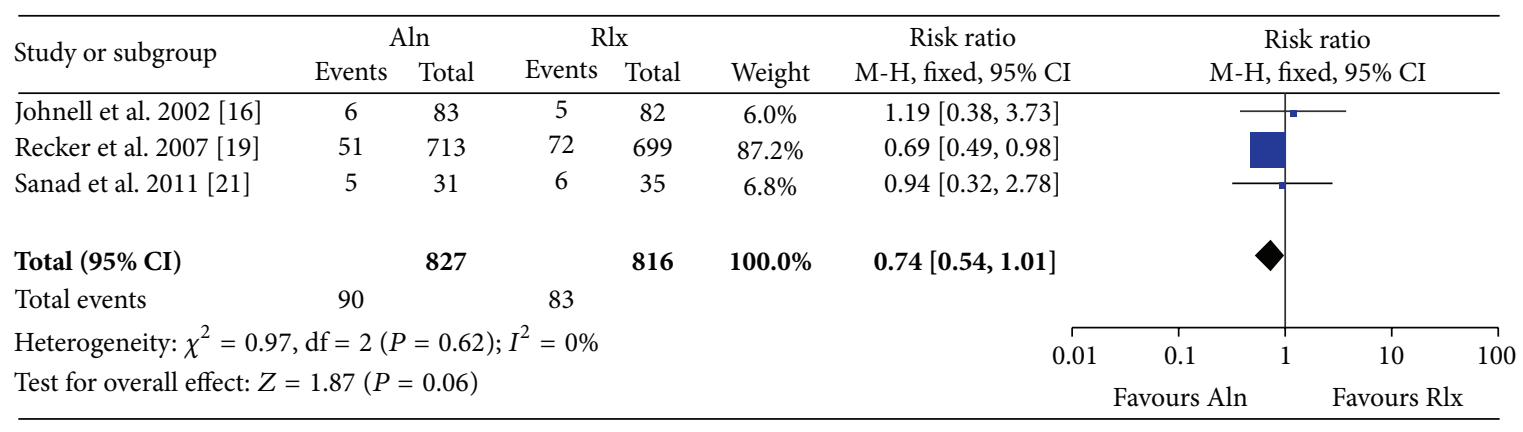

(b)

FIGURE 3: Relative risk of discontinuations due to adverse events, upper gastrointestinal disorders related to treatment, diarrhea events, venous thrombosis events, vasomotor events, and vasodilatation events for postmenopausal women assigned to alendronate compared with raloxifene.

$P=0.01)$, while the difference was not statistical $(P=0.26)$ under the presence of Iwamoto's study [15] (Table 5).

3.7. Meta-Regression Analysis. Women's age, BMI, and pattern of Aln administration have no obvious impacts on the results of fracture (total and nonvertebral fractures) analysis in our metaregression analysis. Though it was insignificant, a widening difference was observed that Aln had more upper GI disorders over Rlx when women's propensity to adopt daily Aln administration or participants' age increased (Supplementary file 1 in the Supplementary Material available online at http://dx.doi.org/10.1155/2013/796510).

3.8. Publication Bias. We found no evidence for publication bias both in vertebral fractures ( 3 trials) and nonvertebral fractures (4 trials), according to both Begg's test and Egger's test [28]. Although the Begg's test funnel plot indicated a potential absence of small size studies which favored Rlx groups in total fractures (6 trials), a trim and fill analysis suggested there were probably 2 missed small trials and the effect size (RR) would be more close to 1 by including them (Supplementary file 2).

\section{Discussion}

Our meta-analysis suggested no superiority of Aln over Rlx in reducing the risk of both vertebral fractures and nonvertebral fractures within a followup of 12-24 months. Aln was more effective in increasing BMD than Rlx. Aln reduced the risk of vasomotor by $57 \%$ but increased the risk of diarrhea by $133 \%$ compared to Rlx. Our subgroup analysis further indicated that the difference between Aln and Rlx in fracture reduction was not materially altered by administration pattern, age, methodological quality, sample size, or industrial funding. The weekly strategy of Aln would further reduce the upper GI disorders and might gain more bone mass increment compared to its daily treatment.

4.1. Strength and Evidence Quality. Our meta-analysis was the first to exclusively comprise head-to-head RCTs, target postmenopausal women and comprehensively evaluate the fracture risk, BMD, and the adverse effects. The previous systematic reviews and the network meta-analyses had indirectly compared the two agents within their multiple agents $[2,6,8]$. Based on the data of the individual agent compared with the placebo, however, their results had poor consistency and great bias due to the variation in the baseline characteristics of participants and the administration pattern of drugs among the trials $[10,11]$. The validity of our findings was further strengthened by strictly following "Cochrane Handbook for systematic Reviews of Interventions 5.0.2” [22]. In particular, we developed the clear criteria of inclusion and exclusion, thoroughly assessed the methodological quality of the included studies, and embarked on the quantitative analysis. Identification of the outlier studies and the sensitivity analysis was to sort out the source of heterogeneity in the present analysis, with the purpose of verifying the results. We also performed the subgroup analysis to comprehensively evaluate the multiple factors potentially influencing the comparative effect. Finally, we used the GRADE system to rigidly assess the quality of evidence, which we aimed to recommend for both agents [24]. Generally, our GRADE analysis showed the evidence of moderate to high quality in most endpoints, which was higher than the previous pooled analyses $[6,12]$ (Table 3).

4.2. Limitations. (a) The combined sample size in current meta-analysis was still limited. However, a large-scale comparative RCT trying to achieve significant fracture prevention difference of the two agents was destined to be infeasible and unnecessary, as the sample size would be unfortunately and unbelievably huge (given the risk fracture of Aln and Rlx in the present analysis was $2.71 \%$ and $2.96 \%$, it would need over 100,000 patients to confirm the theoretical difference of $0.25 \%$ ) [29]. In fact, a well-conducted meta-analysis would always economically and adequately reflect the results of the large-scaled RCT [30]. The present analysis involving all available comparative RCTs with moderate to high quality evidence on the two therapies for postmenopausal women may provide important information for health care providers to supplement the clinical trial evidence. (b) The heterogeneity was detected in the outcome of LS BMD and GI disorder. 


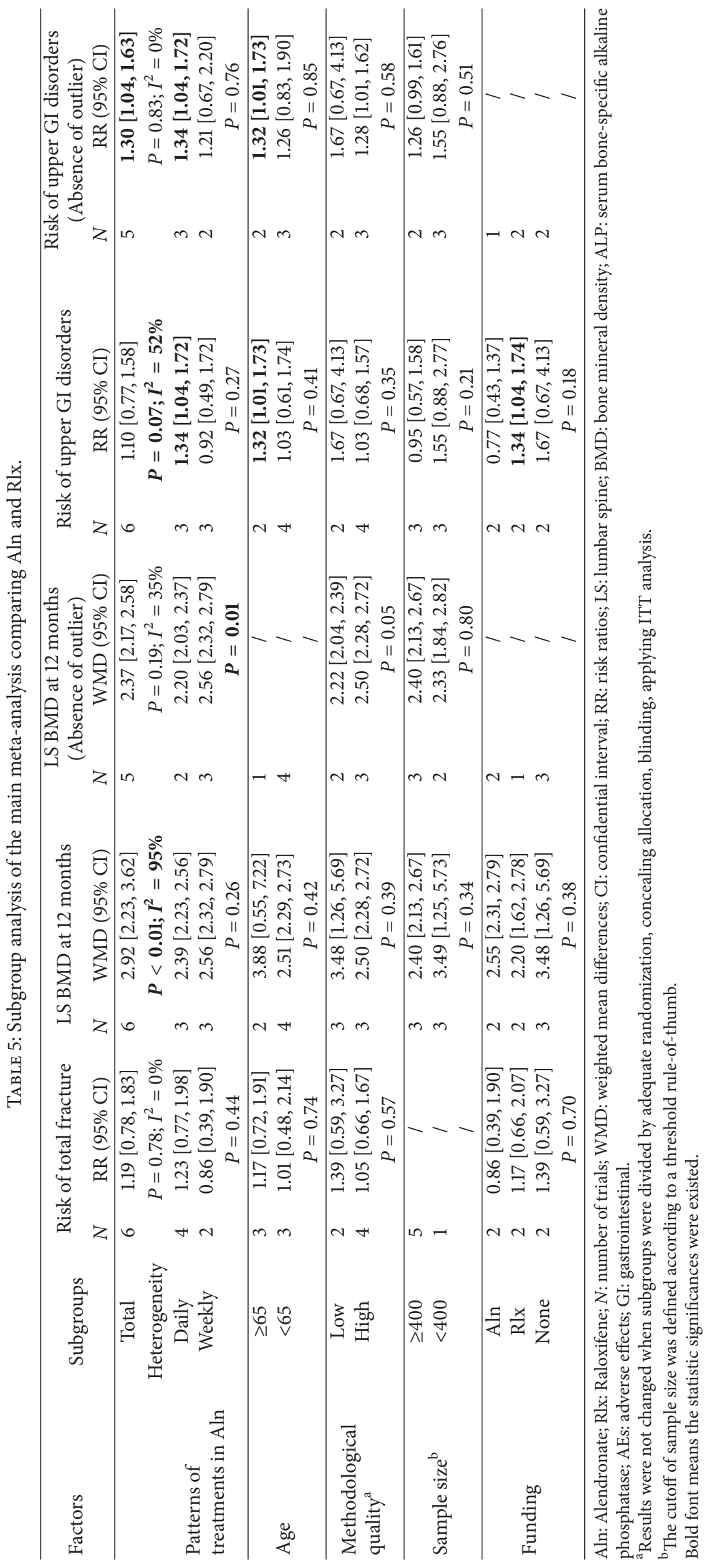


The outlier was identified as Iwamoto et al. [15] in LS BMD and Sambrook et al. [20] in GI disorders. The age of each study (Iwamoto et al.: 69.4, Sambrook et al.: 61.6) which differed from that of the other trials (62.1 67.5) might be the contributor (Table 1). (c) Three studies, lacking adequate randomization, blinding, and concealment of allocation, were considered as the moderate quality $[15,18,21]$. One of them also encountered the loss to followup of certain degree [21]. However, our subgroup analysis suggested the conclusions were not overall influenced by the trial quality. (d) Four studies $[16,17,19,20]$ were sponsored by the pharmaceutical companies related to the either agent. Although the bias of the selective reporting should be considered, the industrial funding was found not to alter the overall results.

4.3. Interpretation and Clinical Implications. Aln was well proved to be more potent than Rlx in inhibition of resorption [31]. Aln could tightly bound to trabecular surfaces where osteoclasts attached and then disrupted their function after its ingestion [32]. As for $\mathrm{Rlx}$, it bound to the raloxifeneestrogen receptor and activated a specific sequence of DNA known as the Raloxifene Responding Element. The subsequent increasing expression of specific cell proteins, which acted as estrogen agonist, resulted in osteoclast suppression [33]. Briefly, the more significant efficacy of Aln over Rlx in BMD increment is probably due to their different pathways of antiremodeling effect $[28,34,35]$.

However, a discrepancy between the statistical difference in bone mass increment and their similar efficacy in vertebral and nonvertebral fracture prevention in the current analysis ought to be cautiously considered. One point should be borne in mind that the BMD decline only partially accounted for the osteoporotic bone fracture. Literatures indicated that the contribution of the increase in BMD accounted for only $4 \%$ of the reduction of vertebral fracture with $\mathrm{Rlx}$ compared with $17 \%$ with Aln [36-39]. Even though Rlx obtained lower bone mass increment, its adequate risk prevention of vertebral fracture has been well established in MORE studies. In addition, Aln allowed fairly accumulation of microdamage in the vertebra, which would be offset by its increase in bone volume though [40], while the positive effect of Rlx on biomechanical properties might adequately cover the inferior bone mass increment, which ultimately bridge the gap in vertebral fracture prevention between both agents.

Currently, Rlx was infrequently prescribed for women with high risk of nonvertebral fractures $[2-4,6,8]$. In the MORE study, Rlx $60 \mathrm{mg} /$ day did not significant decrease nonvertebral fracture (RR: $0.91(0.77,1.07))$ compared with placebo [4]. A recent network meta-analysis performed by Murad et al. also demonstrated Aln other than Rlx achieved a significant reduction in nonvertebral fracture compared to placebo (Aln: odds ratio (OR): 0.78 (0.66, 0.92); Rlx: 0.90 $(0.76,1.03))[6]$. But the inferiority of Rlx under Aln in nonvertebral fractures is still highly inconclusive as the definitive difference was not found in RCTs or systematic reviews. A latest database study of over 100,000 postmenopausal women using inverse probability of treatment weights (IPTWs) method for adjustment highlighted that patients treated with either Rlx or Aln had similar rates in nonvertebral fracture after 8 years of adherent treatment [14]. Our pooled data of head-to-head RCTs also questioned the difference of risk reduction in nonvertebral fractures between both agents.

Patients' adherence to drugs, highly influenced by their tolerance, would substantially affect the benefits of drugs [4143]. Therefore the potential risk of side effects should be thoroughly considered during a decision making. Generally, our review suggested that both drugs were well tolerated with no fatal AEs reported. In particular, Aln increased the incidence of diarrhea while decreased vasomotor events compared to $\mathrm{Rlx}$, which did not require extra medication and seldom caused discontinuation [15]. The increased phlebothrombosis was the main concern for Rlx $[44,45]$. However, in our metaanalysis, only 4 venous thrombosis were found (1/990 in Aln, 3/975 in Rlx), which was really rare. Nevetheless, we agreed that Rlx should be contradicted for postmenopausal women who are at high risk of deep vein thrombosis [46]. It was previously demonstrated that postmenopausal women had a greater propensity to adhere to Rlx and higher satisfaction on drug administration compared with Aln mainly due to more GI disorders associated with $\mathrm{Aln}[47,48]$. In our current analysis, however, the difference of upper GI events and the discontinuation due to AEs between the two agents were balanced. Nevertheless, the greater risk of upper GI disorder of Aln over Rlx was observed when we restricted the analysis to subgroups with daily administration of Aln or subgroups with the age over 65 . The results were confirmed by our metaregression analysis. It implied that the daily Aln other than the weekly Aln increased the frequency of GI irritation. Besides, the aged women had more difficulty in taking Aln properly, which contributed to the more GI symptoms [49, 50]. These results provided some references to improve the compliance of Aln. Although there is not any case reported in the included studies due to the short-term followup, the long term risk of atypical fractures and jaw necrosis with Aln treatment should be under careful surveillance.

\section{Conclusions}

Although the moderate-to-high-quality evidence supported that Aln was more effective in increasing the bone mass than $\mathrm{Rlx}$, the moderate-quality evidence suggested no difference in risk prevention of either vertebral or nonvertebral fractures within a followup of 12-24 months. For Aln the weekly strategy would further reduce the upper GI disorders and might gain more bone mass increment compared to the daily treatment. In addition, more diarrhea episodes but less vasomotor events with Aln should also be considered for enhancing the patient compliance during decision making. Which agent, Aln or Rlx, should be preferred for postmenopausal women remained a patient-oriented matter.

\section{Disclosure}

No sponsors participated in the design and performance of the study, in the collection, analysis, and interpretation of the data, or in the drafting, review, revision, and approval of 
the paper.The datasets extracted from individual papers and meta-analyses are available from the corresponding author.

\section{Ethical Approval}

Not required.

\section{Conflict of Interests}

All authors have completed the Unified Competing Interest form at http://www.icmje.org/coi_disclosure.pdf and declared that the authors and their spouses, partners, or children had no financial relationships with any organisations that might have an interest in the submitted work in the previous three years. No other relationships or activities could appear to have influenced the submitted work.

\section{Authors' Contribution}

All authors contributed to the conceptions of the study and interpretation of data. The study was designed by Tiao Lin, Xun-Zi Cai, and Shi-Gui Yan. Tiao Lin, Zhi-Min Ying Fuzhen Yuan, and Xi Zuo collected data and performed the data analysis. Tiao Lin and Xun-Zi Cai drafted the paper with input from all the authors. The approval of the final version of the paper was given by all the authors. Xun-Zi Cai acts as the guarantor. All the authors had full access to all of the data, including statistical reports and tables in the study and can take responsibility for the integrity of the data and the accuracy of the analysis.

\section{Acknowledgments}

The project was funded by the National Natural Science Foundation of China (81101345, 81101377, and 81171687), the key project of Zhejiang Provincial Department of Science and Technology (2011C13033), the Zhejiang Provincial Natural Science Foundation of China (Y2110239, Y2100161, and Y2090283), the Scientific Research Fund of Zhejiang Provincial Education Department (Y201018936), and the Medical Scientific Research Foundation of Zhejiang Province of China (2012RCA032). All funding sources were independent and had no influence on the study design, the data extraction, analyses, interpretation of the data, writing of this paper, or in the decision to submit the paper for publication.

\section{References}

[1] NIfHaCE N.TA161, Osteoporosis-secondary prevention including strontium ranelate: guidance, 2008.

[2] U. A. Liberman, M. C. Hochberg, P. Geusens et al., "Hip and non-spine fracture risk reductions differ among antiresorptive agents: evidence from randomised controlled trials," International Journal of Clinical Practice, vol. 60, no. 11, pp. 1394-1400, 2006.

[3] P. D. Miller and R. J. Derman, "What is the best balance of benefits and risks among anti-resorptive therapies for postmenopausal osteoporosis?" Osteoporosis International, vol. 21, no. 11, pp. 1793-1802, 2010.
[4] B. Ettinger, D. M. Black, B. H. Mitlak et al., "Reduction of vertebral fracture risk in postmenopausal women with osteoporosis treated with raloxifene: results from a 3-year randomized clinical trial," The Journal of the American Medical Association, vol. 282, no. 7, pp. 637-645, 1999.

[5] D. B. Karpf, D. R. Shapiro, E. Seeman et al., "Prevention of nonvertebral fractures by alendronate: a meta-analysis," The Journal of the American Medical Association, vol. 277, no. 14, pp. 1159-1164, 1997.

[6] M. H. Murad, M. T. Drake, R. J. Mullan et al., "Clinical review. Comparative effectiveness of drug treatments to prevent fragility fractures: a systematic review and network metaanalysis," Journal of Clinical Endocrinology and Metabolism, vol. 97, no. 6, pp. 1871-1880, 2012.

[7] A. Cranney, G. Guyatt, L. Griffith, G. Wells, P. Tugwell, and C. Rosen, "Meta-analyses of therapies for postmenopausal osteoporosis. IX: summary of meta-analyses of therapies for postmenopausal osteoporosis," Endocrine Reviews, vol. 23, no. 4, pp. 570-578, 2002.

[8] C. MacLean, S. Newberry, M. Maglione et al., "Systematic review: comparative effectiveness of treatments to prevent fractures in men and women with low bone density or osteoporosis," Annals of Internal Medicine, vol. 148, no. 3, pp. 197-213, 2008.

[9] H. Raef, M. Al-Bugami, S. Balharith et al., "Updated recommendations for the diagnosis and management of osteoporosis: a local perspective," Annals of Saudi Medicine, vol. 31, no. 2, pp. 111-128, 2011.

[10] F. Song, Y. K. Loke, T. Walsh, A. Glenny, A. J. Eastwood, and D. G. Altman, "Methodological problems in the use of indirect comparisons for evaluating healthcare interventions: survey of published systematic reviews," The British Medical Journal, vol. 338, Article ID b1147, 2009.

[11] L. Trinquart, A. Abbé, and P. Ravaud, "Impact of reporting bias in network meta-analysis of antidepressant placebo-controlled trials," PLoS ONE, vol. 7, no. 4, Article ID e35219, 2012.

[12] N. Freemantle, C. Cooper, A. Diez-Perez et al., "Results of indirect and mixed treatment comparison of fracture efficacy for osteoporosis treatments: a meta-analysis," Osteoporosis International, vol. 24, no. 1, pp. 209-217, 2013.

[13] R. B. Hopkins, R. Goeree, E. Pullenayegum et al., "The relative efficacy of nine osteoporosis medications for reducing the rate of fractures in post-menopausal women," BMC Musculoskeletal Disorders, vol. 12, article 209, 2011.

[14] S. A. Foster, N. Shi, S. Curkendall et al., "Fractures in women treated with raloxifene or alendronate: a retrospective database analysis," BMC Women's Health, vol. 13, article 15, 2013.

[15] J. Iwamoto, Y. Sato, M. Uzawa, T. Takeda, and H. Matsumoto, "Comparison of effects of alendronate and raloxifene on lumbar bone mineral density, bone turnover, and lipid metabolism in elderly women with osteoporosis," Yonsei Medical Journal, vol. 49, no. 1, pp. 119-128, 2008.

[16] O. Johnell, W. H. Scheele, Y. Lu, J. Reginster, A. G. Need, and E. Seeman, "Additive effects of raloxifene and alendronate on bone density and biochemical markers of bone remodeling in postmenopausal women with osteoporosis," Journal of Clinical Endocrinology and Metabolism, vol. 87, no. 3, pp. 985-992, 2002.

[17] M. Luckey, R. Kagan, S. Greenspan et al., "Once-weekly alendronate $70 \mathrm{mg}$ and raloxifene $60 \mathrm{mg}$ daily in the treatment of postmenopausal osteoporosis," Menopause, vol. 11, no. 4, pp. 405-415, 2004.

[18] E. Muscoso, N. Puglisi, C. Mamazza et al., "Antiresorption therapy and reduction in fracture susceptibility in the osteoporotic 
elderly patient: open study," European Review for Medical and Pharmacological Sciences, vol. 8, no. 2, pp. 97-102, 2004.

[19] R. R. Recker, D. Kendler, C. P. Recknor et al., "Comparative effects of raloxifene and alendronate on fracture outcomes in postmenopausal women with low bone mass," Bone, vol. 40, no. 4, pp. 843-851, 2007.

[20] P. N. Sambrook, P. Geusens, C. Ribot et al., "Alendronate produces greater effects than raloxifene on bone density and bone turnover in postmenopausal women with low bone density: results of EFFECT (EFficacy of FOSAMAX versus EVISTA Comparison Trial) International," Journal of Internal Medicine, vol. 255, no. 4, pp. 503-511, 2004.

[21] Z. Sanad, H. Ellakwa, and B. Desouky, "Comparison of alendronate and raloxifene in postmenopausal women with osteoporosis," Climacteric, vol. 14, no. 3, pp. 369-377, 2011.

[22] J. P. T. Higgins, S. Green, and Cochrane Collaboration, Cochrane Handbook for Systematic Reviews of Interventions, WileyBlackwell, Chichester, UK, 2008.

[23] B. Baujat, C. Mahé, J. Pignon, and C. Hill, "A graphical method for exploring heterogeneity in meta-analyses: application to a meta-analysis of 65 trials," Statistics in Medicine, vol. 21, no. 18, pp. 2641-2652, 2002.

[24] D. Atkins, D. Best, P. A. Briss et al., "Grading quality of evidence and strength of recommendations," The British Medical Journal, vol. 328, no. 7454, pp. 1490-1494, 2004.

[25] J. D. Adachi, S. Adami, P. M. Kulkarni, M. Wong, and J. L. Stock, "Similar proportions of women lose bone mineral density with raloxifene or alendronate treatment," Journal of Clinical Densitometry, vol. 8, no. 3, pp. 273-277, 2005.

[26] R. Eastell, A. Rogers, X. Ni, and J. H. Krege, "Effects of raloxifene and alendronate on bone turnover as assessed by procollagen type I N-terminal propeptide," Osteoporosis International, vol. 22, no. 6, pp. 1927-1934, 2011.

[27] B. Yanik, N. Bavbek, T. Yanğk et al., "The effect of alendronate, risedronate, and raloxifene on renal functions, based on the cockcroft and gault method, in postmenopausal women," Renal Failure, vol. 29, no. 4, pp. 471-476, 2007.

[28] M. R. Allen and D. B. Burr, "Three years of alendronate treatment results in similar levels of vertebral microdamage as after one year of treatment," Journal of Bone and Mineral Research, vol. 22, no. 11, pp. 1759-1765, 2007.

[29] J. A. Kanis, A. Oden, O. Johnell et al., "Uncertain future of trials in osteoporosis," Osteoporosis International, vol. 13, no. 6, pp. 443-449, 2002.

[30] J. LeLorier, G. Grégoire, A. Benhaddad, J. Lapierre, and F. Derderian, "Discrepancies between meta-analyses and subsequent large randomized, controlled trials," The New England Journal of Medicine, vol. 337, no. 8, pp. 536-542, 1997.

[31] M. Giner, M. J. Rios, M. J. Montoya, M. A. Vázquez, C. Miranda, and R. Pérez-Cano, "Alendronate and raloxifene affect the osteoprotegerin/RANKL system in human osteoblast primary cultures from patients with osteoporosis and osteoarthritis," European Journal of Pharmacology, vol. 650, no. 2-3, pp. 682687, 2011.

[32] S. Takeyama and H. Shinoda, "Pharmacological actions and pharmacokinetics of bisphosphonates," Clinical Calcium, vol.13, pp. 115-121, 2003.

[33] J. R. Rey, E. V. Cervino, M. L. Rentero, E. C. Crespo, A. O. Alvaro, and M. Casillas, "Raloxifene: mechanism of action, effects on bone tissue, and applicability in clinical traumatology practice," Open Orthopaedics Journal, vol. 3, pp. 14-21, 2009.
[34] M. R. Allen, K. Iwata, M. Sato, and D. B. Burr, "Raloxifene enhances vertebral mechanical properties independent of bone density," Bone, vol. 39, no. 5, pp. 1130-1135, 2006.

[35] M. R. Allen and D. B. Burr, "Bisphosphonate effects on bone turnover, microdamage, and mechanical properties: what we think we know and what we know that we don't know," Bone, vol. 49, no. 1, pp. 56-65, 2011.

[36] S. R. Cummings, D. B. Karpf, F. Harris et al., "Improvement in spine bone density and reduction in risk of vertebral fractures during treatment with antiresorptive drugs," The American Journal of Medicine, vol. 112, no. 4, pp. 281-289, 2002.

[37] Z. Li, M. P. Meredith, and M. S. Hoseyni, "A method to assess the proportion of treatment effect explained by a surrogate endpoint," Statistics in Medicine, vol. 20, no. 21, pp. 3175-3188, 2001.

[38] S. Sarkar, B. H. Mitlak, M. Wong, J. L. Stock, D. M. Black, and K. D. Harper, "Relationships between bone mineral density and incident vertebral fracture risk with raloxifene therapy," Journal of Bone and Mineral Research, vol. 17, no. 1, pp. 1-10, 2002.

[39] N. B. Watts, C. Cooper, R. Lindsay et al., "Relationship between changes in bone mineral density and vertebral fracture risk associated with risedronate: greater increases in bone mineral density do not relate to greater decreases in fracture risk," Journal of Clinical Densitometry, vol. 7, no. 3, pp. 255-261, 2004.

[40] M. R. Allen, K. Iwata, R. Phipps, and D. B. Burr, "Alterations in canine vertebral bone turnover, microdamage accumulation, and biomechanical properties following 1-year treatment with clinical treatment doses of risedronate or alendronate," Bone, vol. 39, no. 4, pp. 872-879, 2006.

[41] J. R. Curtis, E. Delzell, L. Chen et al., “The relationship between bisphosphonate adherence and fracture: is it the behavior or the medication? Results from the placebo arm of the fracture intervention trial," Journal of Bone and Mineral Research, vol. 26, no. 4, pp. 683-688, 2011.

[42] I. Imaz, P. Zegarra, J. González-Enríquez, B. Rubio, R. Alcazar, and J. M. Amate, "Poor bisphosphonate adherence for treatment of osteoporosis increases fracture risk: systematic review and meta-analysis," Osteoporosis International, vol. 21, no. 11, pp. 1943-1951, 2010.

[43] A. R. Patrick, M. A. Brookhart, E. Losina et al., "The complex relation between bisphosphonate adherence and fracture reduction," Journal of Clinical Endocrinology and Metabolism, vol. 95, no. 7, pp. 3251-3259, 2010.

[44] E. Barrett-Connor, L. Mosca, P. Collins et al., "Effects of raloxifene on cardiovascular events and breast cancer in postmenopausal women," The New England Journal of Medicine, vol. 355, no. 2, pp. 125-137, 2006.

[45] E. Barrett-Connor, J. A. Cauley, P. M. Kulkarni, A. Sashegyi, D. A. Cox, and M. J. Geiger, "Risk-benefit profile for raloxifene: 4-year data from the multiple outcomes of raloxifene evaluation (MORE) randomized trial," Journal of Bone and Mineral Research, vol. 19, no. 8, pp. 1270-1275, 2004.

[46] H. Hansdóttir, "Raloxifene for older women: a review of the literature," Clinical Interventions in Aging, vol. 3, no. 1, pp. 45-50, 2008.

[47] P. D. Miller, G. Woodson, A. A. Licata et al., "Rechallenge of patients who had discontinued alendronate therapy because of upper gastrointestinal symptoms," Clinical Therapeutics, vol. 22, no. 12, pp. 1433-1442, 2000.

[48] E. G. Pasion, S. K. Sivananthan, A. W. Kung et al., "Comparison of raloxifene and bisphosphonates based on adherence 
and treatment satisfaction in postmenopausal Asian women," Journal of Bone and Mineral Metabolism, vol. 25, no. 2, pp. 105113, 2007.

[49] J. D. Adachi, R. Y. Faraawi, M. F. J. O’Mahony et al., “Upper gastrointestinal tolerability of alendronate sodium monohydrate $10 \mathrm{mg}$ once daily in postmenopausal women: a 12-week, randomized, double-blind, placebo-controlled, exploratory study," Clinical Therapeutics, vol. 31, no. 8, pp. 1747-1753, 2009.

[50] B. Ettinger, A. Pressman, and J. Schein, "Clinic visits and hospital admissions for care of acid-related upper gastrointestinal disorders in women using alendronate for osteoporosis," The American Journal of Managed Care, vol. 4, no. 10, pp. 1377-1382, 1998. 


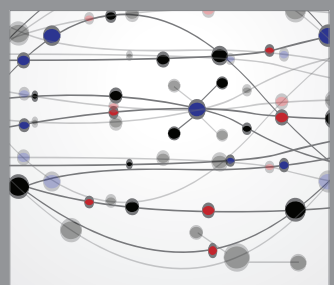

The Scientific World Journal
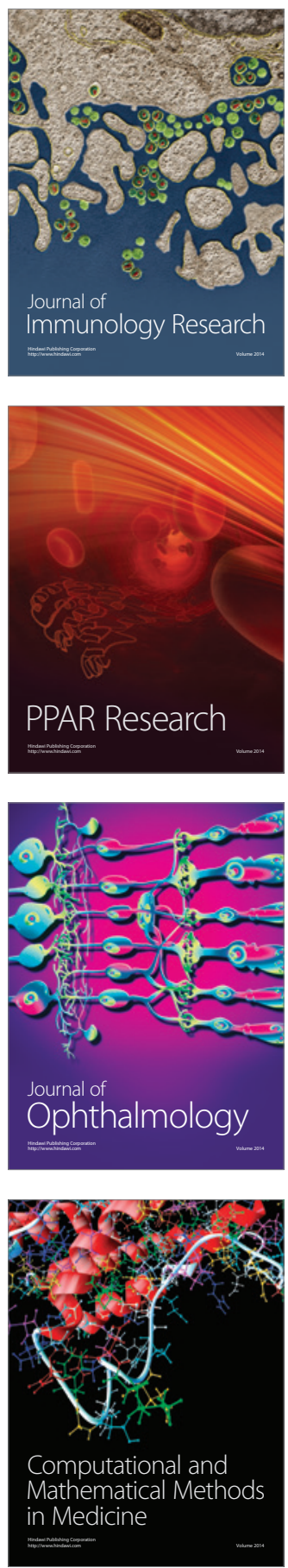

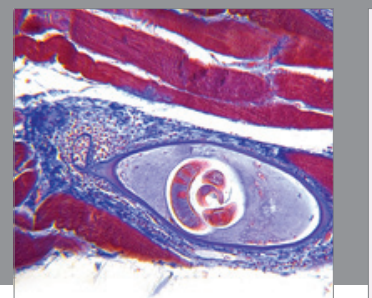

Gastroenterology

Research and Practice
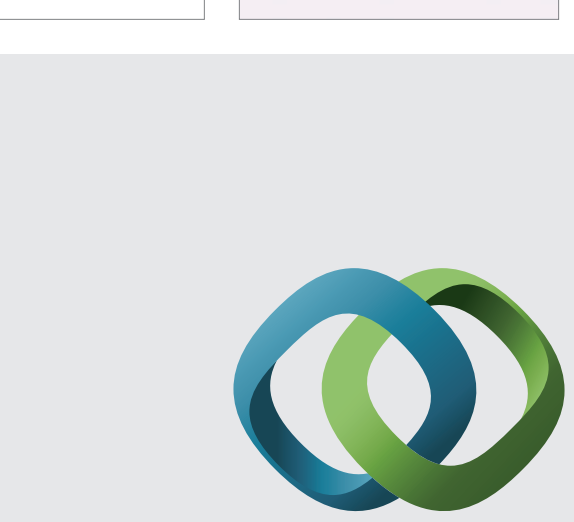

\section{Hindawi}

Submit your manuscripts at

http://www.hindawi.com
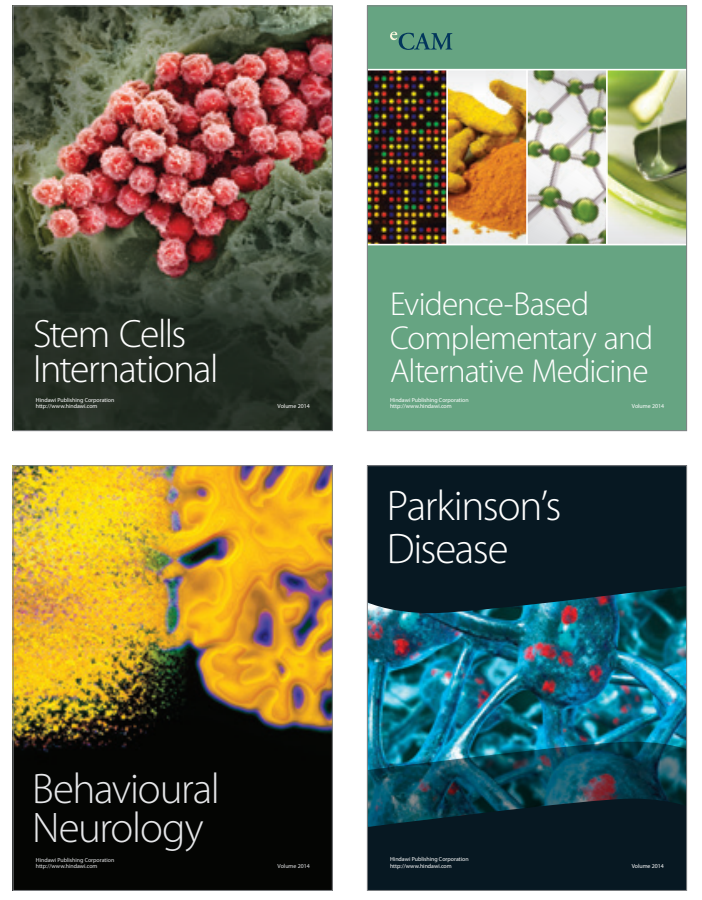
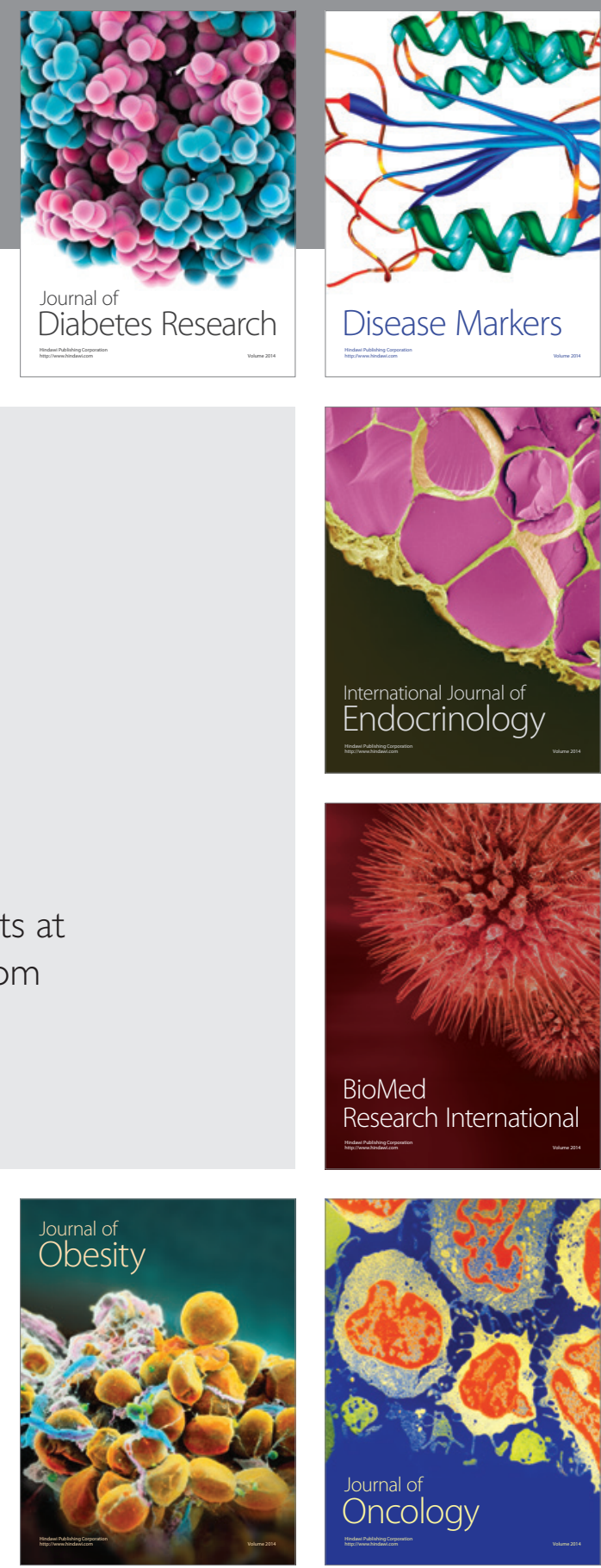

Disease Markers
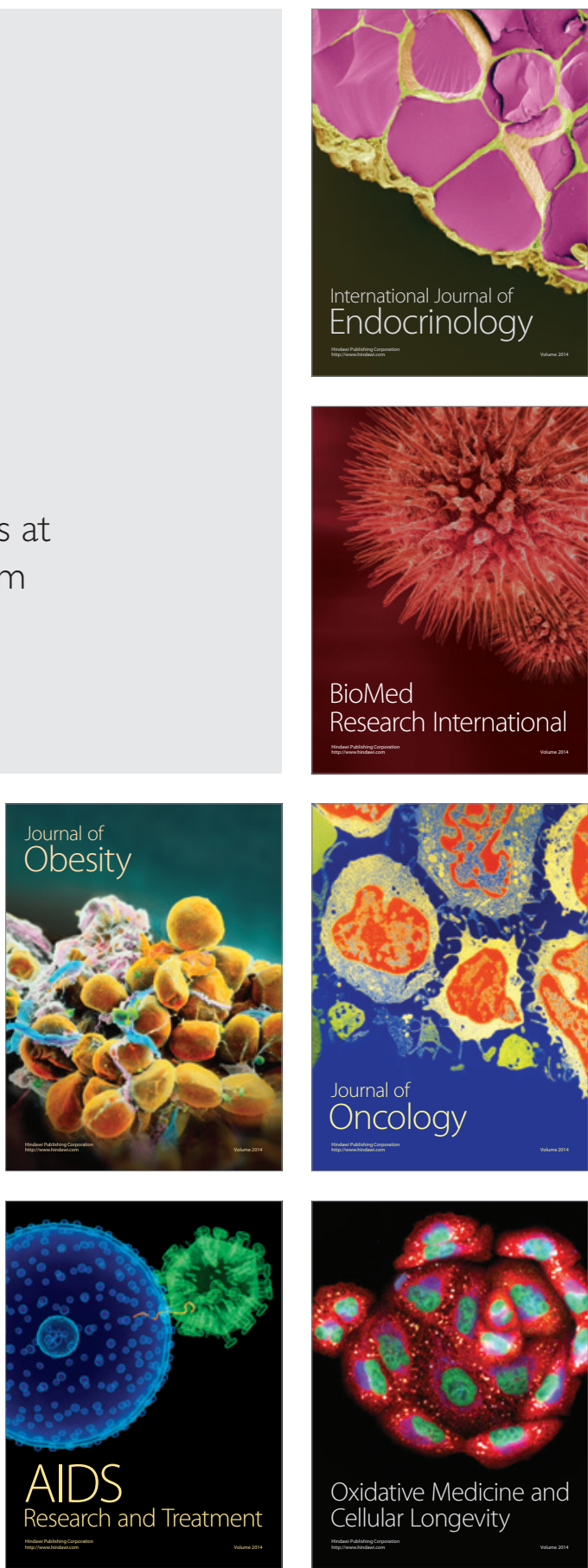